\title{
MASONRY MICRO-MODELLING ADOPTING A DISCONTINUOUS FRAMEWORK
}

\author{
J. Pina-Henriques and Paulo B. Lourenço \\ School of Engineering, University of Minho, Guimarães, Portugal
}

\begin{abstract}
Several continuous and discontinuous micro-modelling approaches for masonry assemblages are currently under investigation by the authors. The aim is to find a model that provides a suitable description of the material behaviour under compression. Firstly, the behaviour under short-term loading is being considered and, in a later stage, also long-term loading will be addressed. The present paper illustrates the recent advances in the research, focused on the assessment and application of a proposed discontinuous model to the simulation of uniaxial compression tests of masonry prisms. In this model, a fictitious micro-structure composed by linear elastic particles separated by non-linear interfaces is adopted to model units and mortar. The main results obtained and their critical discussion are given in the paper.
\end{abstract}

Keywords: ancient masonry structures, non-linear finite-element analysis, micromodelling strategies, interface elements.

\section{Introduction}

Micro-modelling of masonry, understood as a computational strategy where both units and mortar are detailed, is a powerful tool in the analysis of this composite material. This type of approach takes the analysis down to the components level, allowing understanding of the basic phenomena that occur in masonry upon increasing loading. The authors are currently addressing both continuous and discontinuous approaches, attempting to identify a model that is able to predict accurately the behaviour of masonry under compression. Firstly, short-term loading is considered and, in a later stage, long-term loading will be also accounted for.

The results obtained using a non-linear continuous micro-model have been reported elsewhere, see Pina-Henriques and Lourenço [1], and will be only briefly reviewed here. In [1] it is shown that continuum micro-models largely overestimate 
the experimental strength of masonry prisms under uniaxial compression. Values ranging from 160 to $180 \%$ for the ratio between the predicted strength and the experimental strength were found. Alternative modelling approaches seem, thus, to be needed. The present paper illustrates the recent advances in the research, regarding the application of a discontinuous model to simulate a fictitious microstructure given to the units and mortar.

\section{Model concept}

\subsection{Background of the model}

Several advanced computational approaches are currently available to deal with the particulate nature of materials such as soils, rocks, concrete or masonry. Within the most common ones, two main types of analysis can be distinguished: discrete element methods and the finite element method (FEM) including interface elements.

The micro-model here proposed is developed under a FEM framework, where the discontinuous nature of the masonry components is taken into account. A fictitious micro-structure is given to units and mortar, which is composed by linear elastic polygons (here named particles) separated by non-linear interface elements, see Figure 1. All the inelastic phenomena occur in the interface elements and the process of fracturing consists of progressive bond-breakage.

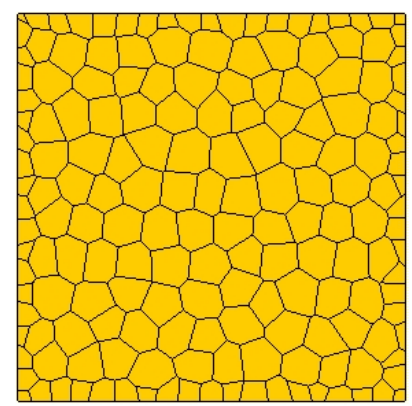

Figure 1: Fictitious micro-structure given to units and mortar.

Three nodded plane stress triangular elements with Gauss integration were utilized in this study to model the particles. For the interfaces, line interface elements with zero thickness were adopted. A high dummy stiffness was given to the interface elements to avoid interpenetration of the particles, as it is clear that the amount of penetration will be higher with decreasing interface stiffness. Values ranging from $1 \times 10^{4}$ to $8 \times 10^{4} \mathrm{~N} / \mathrm{mm}^{3}$ were chosen so that overlapping of neighbouring particles would not become visible. Schellekens (1992) reported that beyond stiffness values of $1 \times 10^{3} \mathrm{~N} / \mathrm{mm}^{3}$ the application of the Gauss integration scheme leads to oscillatory results. To overcome such deficiency, a three-point Lobatto integration scheme was used.

The dilatancy angle measures the uplift upon shearing. The analyses here reported were performed in a non-associated plasticity context, assuming a dilatancy equal to zero. In such way, a particle can slide over the other without producing any 
normal displacement. Non-zero dilatancy associated with the boundary conditions adopted (see Sections 3 and 4) could induce high normal stresses and locking of the particles, resulting in increasing brittleness and strength. Also for unit-mortar interfaces, Lourenço [3] recommends a value of zero for the dilatancy angle.

The constitutive model used for the interface elements was formulated by Lourenço [3] and is implemented in the adopted finite element code Diana [4, 5]. This model includes a tension cut-off for tensile failure (mode I), a Coulomb friction envelope for shear failure (mode II) and a cap mode for compressive failure. Softening is present in all three modes and is preceded by hardening in the case of the cap mode.

\subsection{Mesh construction}

A computer routine has been written in Visual Basic 6.0 (VB 6.0) to generate the mesh, see Figure 2. The input data that must be given to the routine are the boundaries of the surface to mesh, the average size of the particles and a distortion factor $D F$, which randomly displaces the particles from their original positions, see Figure 3 . In addition, the type and average size of the finite elements utilized must be specified. The discretization of the continuum into particles is based in the Voronoi diagram. To obtain the coordinates of the vertices of the Voronoi regions, the routine executes a call to an external freeware DOS program named Qhull [6] and then processes the output data.

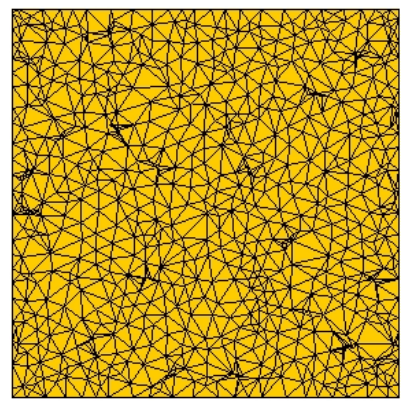

(a)

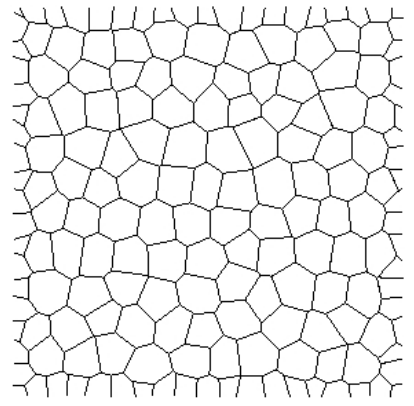

(b)

Figure 2: Finite element mesh: (a) particles mesh and (b) interfaces mesh.

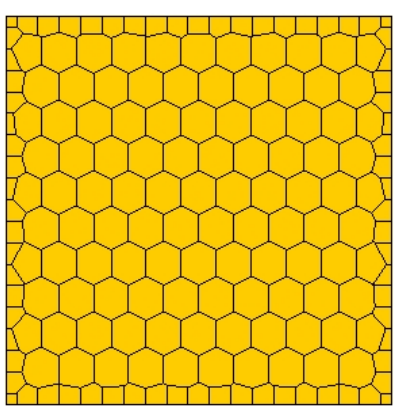

(a)

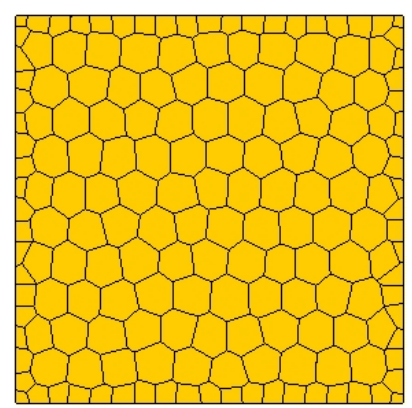

(b)

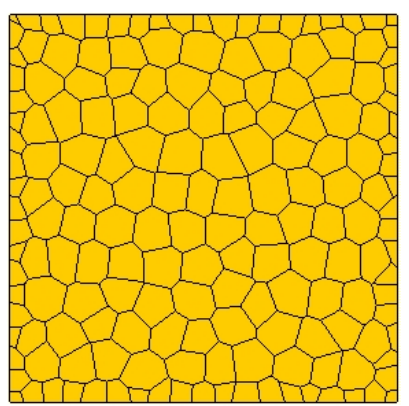

(c)

Figure 3: Models with different distortion factors $D F$ : (a) $D F=0$, (b) $D F=0.3$ and (c) $D F=0.6$. 


\subsection{Material heterogeneity}

In heterogeneous materials the disorder of the material properties at the micro-level is a key issue in the fracture process. The material disorder was given to the model by attributing to each particle and to each interface random material parameters. For that purpose, a VB 6.0 routine was written to generate Gaussian distributions of the modulus of elasticity of the particles and of the strength parameters of the interfaces. As input data, the average values of the parameters, the corresponding coefficients of variation and the number of different values to generate are required.

\section{Model response. Elementary tests}

\subsection{Compressive uniaxial behaviour}

To assess the uniaxial compressive behaviour of the model, a specimen with dimensions $100 \times 100 \times 100 \mathrm{~mm}^{3}$ was simulated under pure uniaxial compression. Three different values for the distortion factor $D F$ were considered and, for each one, three different levels of mesh refinement $R L$ were assumed. Given the random nature of the model, for each combination $D F-R L$, three analyses were performed with different generated meshes. The simulations were carried out using plain stress elements and under symmetry conditions. In this way, the boundaries remain straight during the analyses, aiming at reproducing macro homogeneous boundary conditions. Thus, specimens are considered as part of a larger portion.

The material properties given to the particles and interfaces are characterized by an average value and a coefficient of variation $C V$. The parameters are kept constant for all specimens, so that the influence of the geometry can be assessed. The elastic properties attributed to the particles (elastic modulus $E$ and coefficient of Poisson $v$ ) and to the interfaces (normal modulus $k_{n}$ and shear modulus $k_{s}$ ) are given in Table 1. The inelastic properties of the interfaces are shown in Table 2. Here, $f_{t}$ is the tensile strength, $G_{f I}$ is the mode I fracture energy, $c$ is the cohesion, $G_{f I I}$ is the mode II fracture energy and $\tan \phi$ is the friction coefficient.

\begin{tabular}{|c|c|c|c|}
\hline & & Average values & $C V[\%]$ \\
\hline \multirow{2}{*}{ Particles } & $E$ & $5000 \mathrm{~N} / \mathrm{mm}^{2}$ & 30 \\
\cline { 2 - 4 } & $v$ & 0.15 & 0 \\
\hline \multirow{2}{*}{ Interfaces } & $k_{n}$ & $10^{4} \mathrm{~N} / \mathrm{mm}^{3}$ & 0 \\
\cline { 2 - 4 } & $k_{s}$ & $10^{4} \mathrm{~N} / \mathrm{mm}^{3}$ & 0 \\
\hline
\end{tabular}

Table 1: Elastic properties for the particles and interfaces.

\begin{tabular}{|c|c|c|}
\hline & Average values & $C V[\%]$ \\
\hline$f_{t}$ & $1.0 \mathrm{~N} / \mathrm{mm}^{2}$ & 50 \\
\hline$G_{f I}$ & $0.050 \mathrm{~N} / \mathrm{mm}$ & 50 \\
\hline$c$ & $1.5 \mathrm{~N} / \mathrm{mm}^{2}$ & 50 \\
\hline$G_{f I I}$ & $0.75 \mathrm{~N} / \mathrm{mm}$ & 50 \\
\hline $\tan \phi$ & 0.30 & 50 \\
\hline
\end{tabular}

Table 2: Inelastic properties for the interfaces. 
A unitary value was assumed for the tensile strength and the cohesion was obtained according to $c=1.5 f_{t}$. This relation was proposed by Lourenço [7] for unit - mortar interfaces. For $G_{f f}$, a value in agreement with the results obtained by Van der Pluijm [8] was adopted and for $G_{f I I}$ a value about five times higher the value proposed by Lourenço [7] for unit - mortar interfaces $(0.1 c)$ was used. The friction coefficient was chosen so that the ratio between the compressive and tensile strengths of the element simulated was about ten, which is a ratio often found for masonry units, see Schubert [8]. Given the fact that the approach here followed is phenomenological and not physical, the values adopted for the coefficient of variation of the different material parameters are not related with their experimental variability but were chosen so that the overall response of the model resembles the experimental behaviour.

Typical numerical stress-strain diagrams obtained for each type of geometry are given in Figure 4. The behaviour observed shows that increasing distortion of the particles leads to decreasing brittleness. There seems to be also a relation between brittleness and the mesh refinement. In fact, specimens with a refinement level $n$ show a more brittle behaviour, characterized by sudden load drops, than specimens with refinement levels $2 n$ and $4 n$. However, it is noted that there are not much difference in the response beyond a level of refinement of $2 n$.

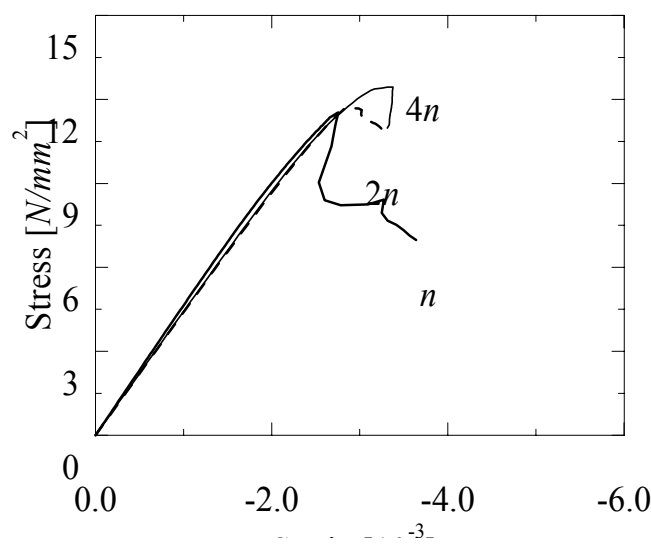

(a)

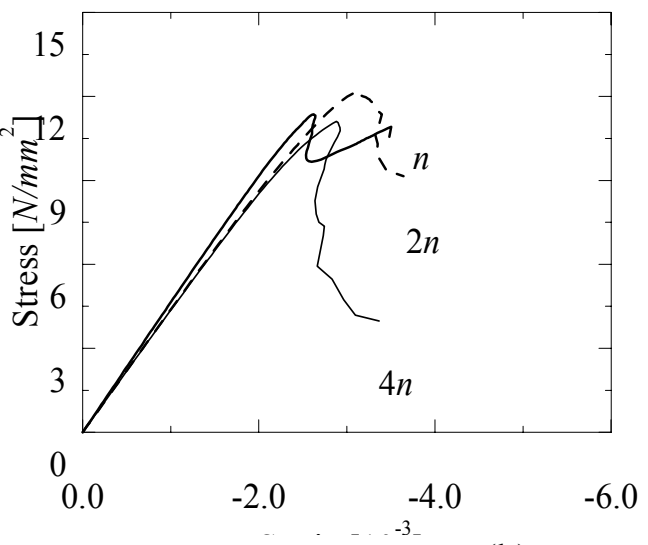

(b)

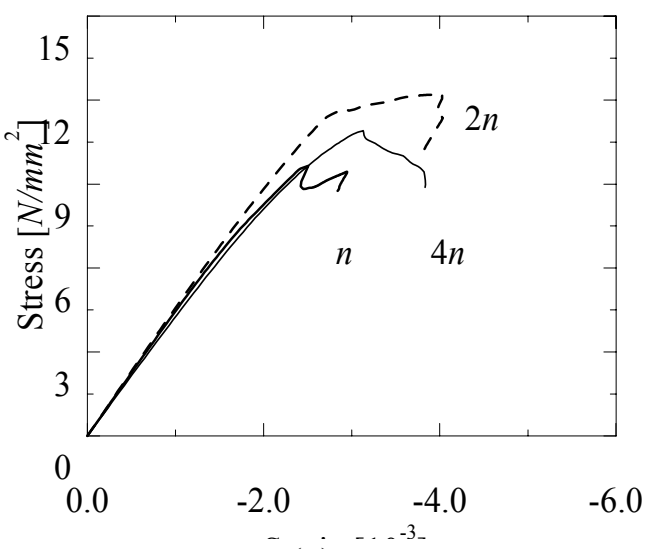

(c)

Figure 4: Typical stress-strain diagrams for different levels of mesh refinement $(n, 2 n, 4 n)$ and different distortion factors: (a) $D F=0$, (b) $D F=0.3$, (c) $D F=0.6$. 
Table 3 gives the values for the compressive strength $f_{c}$ obtained with the simulations, according to the distortion factor and level of mesh refinement. In brackets, the values for the coefficient of variation are given. There is an increase of the variability of the strength values with increasing distortion of the particles and with decreasing refinement of the mesh. The variation obtained, considering that a different mesh was generated for each analysis, fairly reproduces, as expected, the experimental variability of results. Moreover, it can be observed that the strength values have a slightly decreasing trend with increasing distortion, especially for lower levels of mesh refinement. Nevertheless, the average values for $2 n$ and $4 n$ can be considered as mesh size and mesh distortion independent for practical purposes.

\begin{tabular}{|c|c|c|c|}
\hline & $R L=n$ & $R L=2 n$ & $R L=4 n$ \\
\hline$D F=0$ & $12.0(10.4 \%)$ & $11.6(0.6 \%)$ & $12.0(6.7 \%)$ \\
\hline$D F=0.3$ & $10.9(15.4 \%)$ & $11.1(10.4 \%)$ & $11.6(3.3 \%)$ \\
\hline$D F=0.6$ & $9.9(15.3 \%)$ & $11.0(13.8 \%)$ & $11.4(8.4 \%)$ \\
\hline
\end{tabular}

Table 3: Average values from three analyses obtained for the compressive strength $f_{c}\left[\mathrm{~N} / \mathrm{mm}^{2}\right]$ according to different levels of mesh refinement $R L$ and distortion factors $D F$ (values in brackets give the coefficient of variation $C V$ ).

The failure patterns of the specimens are depicted in Figure 5 in terms of deformed meshes. From Figure 5 it is clear that under certain combinations of distortion and refinement of the mesh, the failure patterns become too dependent of the mesh configuration. For instance, in $D F=0$ and $R L=4 n$, the crack pattern denotes a clear diagonal tendency, unlike, for example, $D F=0.6$ and $R L=4 n$ where the crack pattern resembles experienced compression crack patterns, with predominant vertical cracks. In conclusion, the mesh configuration appears to have a larger influence in the crack patterns of numerical specimens with lower distortion factors and with more refined meshes. Nevertheless, the value of the failure load is not affected by the mesh preferential orientation.

A comparative evaluation between the contribution of the interfaces tensile and shear parameters on the compressive strength of the specimens was also performed. To achieve this purpose, compression simulations assuming three different values for the interfaces tensile strength were assumed while the model shear parameters were kept constant. The results obtained are given in Table 4 and the stress-strain diagrams are illustrated in Figure 6. As expected, a decreasing trend for the compressive strength with decreasing tensile strength was found. However, the reduction of strength obtained is relatively small when compared with the tensile strength reduction. Such response shows that the compressive failure of the proposed particulate model is mainly governed by the parameters describing the interfaces shear behaviour. 


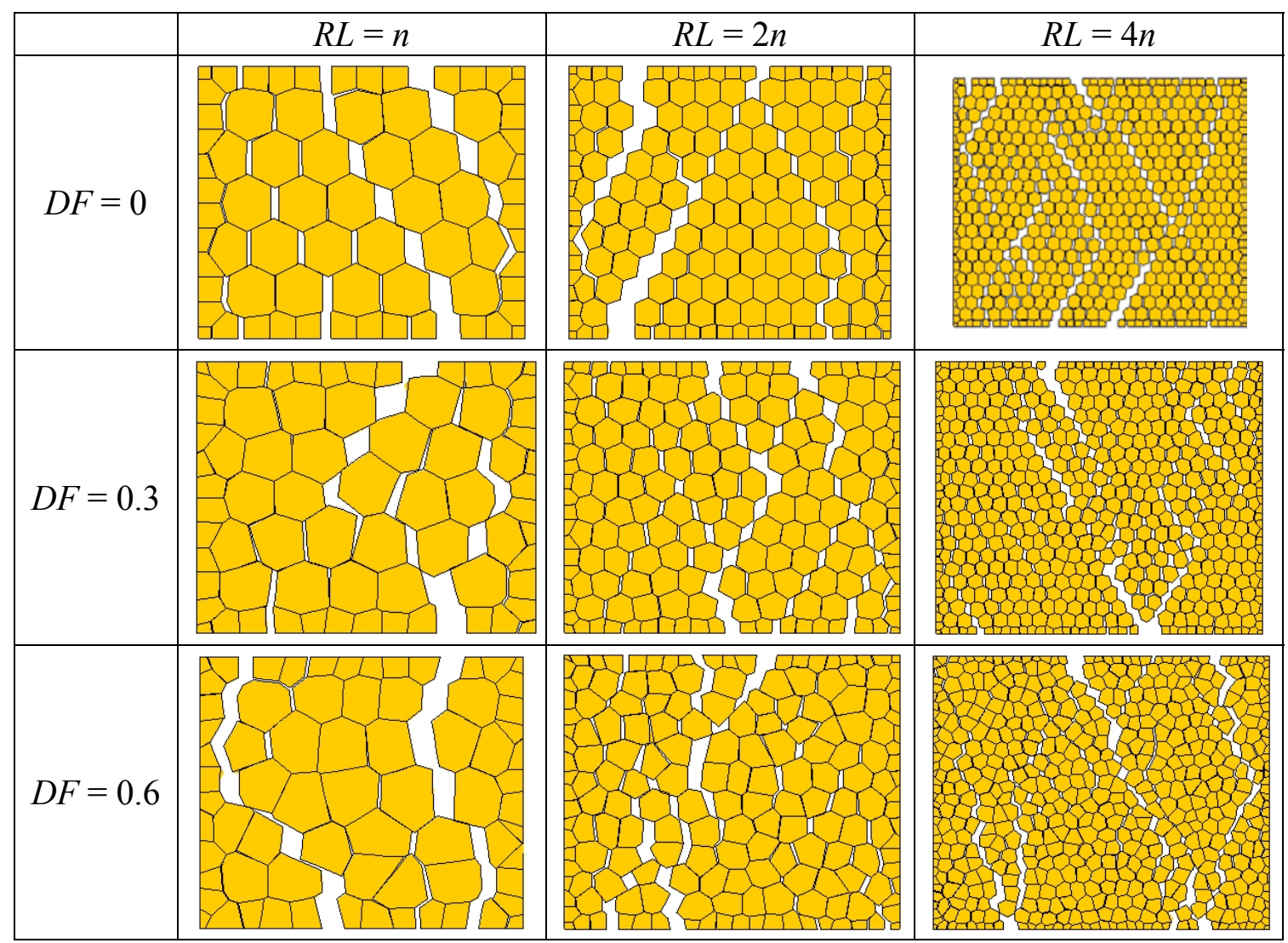

Figure 5: Typical deformed meshes obtained from the compression simulations. Three distortion factors $D F$ and mesh refinement levels $R L$ were considered.

\begin{tabular}{|c|c|c|}
\hline & $f_{c}\left[\mathrm{~N} / \mathrm{mm}^{2}\right]$ & $c / f_{t}[-]$ \\
\hline$f_{t}=1.0 \mathrm{~N} / \mathrm{mm}^{2}$ & 11.1 & 1.5 \\
\hline$f_{t}=0.50 \mathrm{~N} / \mathrm{mm}^{2}$ & 10.0 & 3.0 \\
\hline$f_{t}=0.25 \mathrm{~N} / \mathrm{mm}^{2}$ & 9.2 & 6.0 \\
\hline
\end{tabular}

Table 4: Values for the material element compressive strength $f_{c}$ for different values of interfaces tensile strength $f_{t}$.

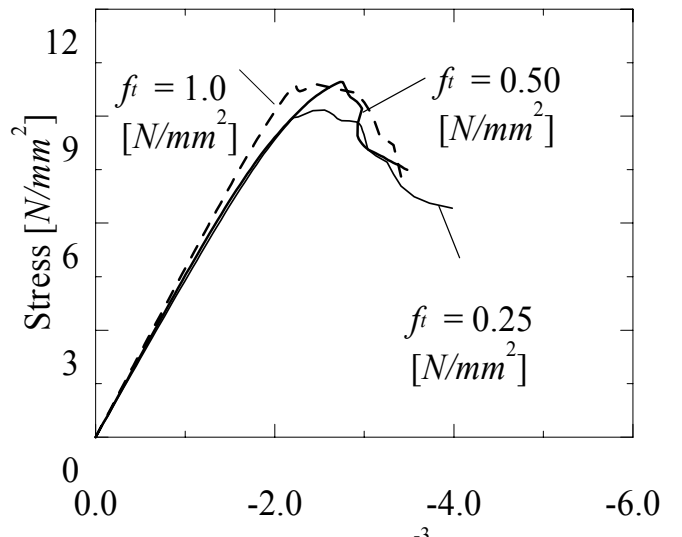

Figure 6: Stress-strain compression diagrams for specimens with $D F=0.3$ and $R L=$ $2 n$, considering three different values for the interfaces tensile strength $f_{t}$. 


\subsection{Tensile uniaxial behaviour}

The same specimens and the same material properties used for the compression simulations, as described in Section 3.1, were used to evaluate the tensile behaviour of the model. The values obtained for the specimens tensile strength, according to the level of mesh refinement and the distortion factor, are given in Table 5.

Slightly decreasing values for the tensile strength were found for increasing values of the distortion factor. However, as for compression, the average values can be considered as mesh size and mesh distortion independent for practical purposes. Moreover, it is noted that increasing mesh refinement is accompanied by decreasing variability of the strength values and rather low values are obtained for $R L=4 n$.

\begin{tabular}{|c|c|c|c|}
\hline & $R L=n$ & $R L=2 n$ & $R L=4 n$ \\
\hline$D F=0$ & $1.1(11.8 \%)$ & $1.1(9.8 \%)$ & $1.1(4.1 \%)$ \\
\hline$D F=0.3$ & $0.9(9.8 \%)$ & $1.0(7.6 \%)$ & $1.1(4.1 \%)$ \\
\hline$D F=0.6$ & $0.9(13.3 \%)$ & $1.0(1.1 \%)$ & $1.0(1.4 \%)$ \\
\hline
\end{tabular}

Table 5: Average values from three analyses obtained for the compressive strength $f_{c}\left[\mathrm{~N} / \mathrm{mm}^{2}\right]$ according to different levels of mesh refinement $R L$ and distortion factors $D F$ (values in brackets give the coefficient of variation $C V$ ).

Three typical types of tensile response were obtained from the model. Figure 7 illustrates the responses for specimens with similar geometries and material properties. Each type of response is associated with a different failure pattern, which is depicted in Figure 8. Depending on the failure pattern, the specimen mode I fracture energy can range from a value similar to the mode I fracture energy given to the interface elements to an almost infinite value due to a high residual tensile strength. Such residual strength develops when diagonal cracks appear, originating friction between the particles due to the imposed boundary (symmetry) conditions.

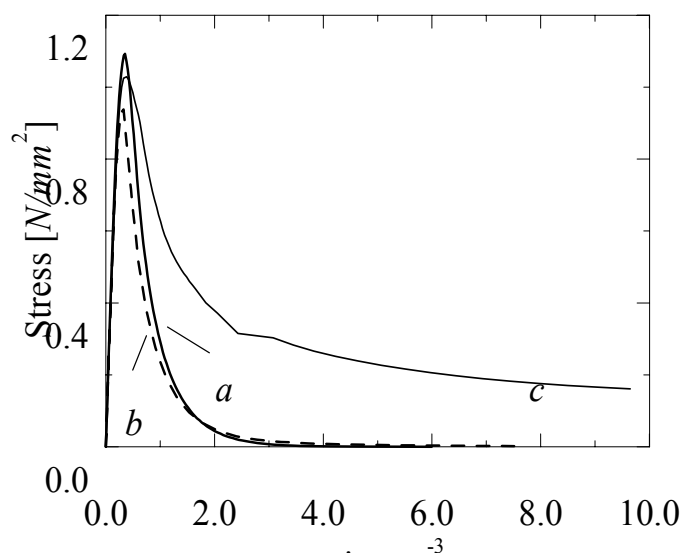

Figure 7: Tensile stress-strain diagrams obtained for $R L=2 n$ and $D F=0.3$. Figure 8 illustrates the failure pattern associated to each diagram. 

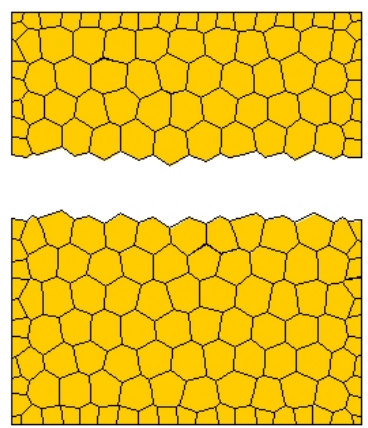

(a)
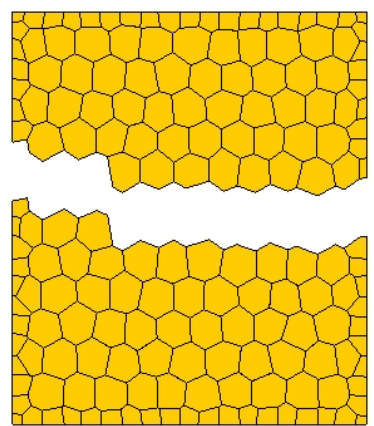

(b)

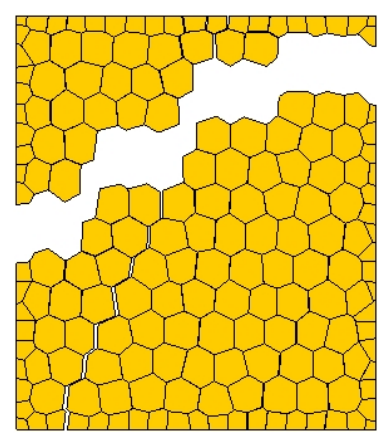

(c)

Figure 8: Deformed meshes for specimens with a mesh refinement level of $2 n$ and a distortion factor equal to 0.3 , illustrating three different types of tensile failure.

\subsection{Size effect}

The effect of size is an important issue when estimating the strength or stress-strain relation of quasibrittle materials. It is now well known that tensile failure occurs with strain localization causing the response to be dependent on the size of the element. Recently, researchers have shown that also for concrete elements loaded in compression, failure occurs with strain localization although in a wider region and with a failure mechanism rather more complex than tensile failure. Experiments carried out have shown that compressive strength and post-peak ductility tend to increase with decreasing size of the element, see Vonk [10] and Kim and Yi [11].

The ability of the micro-model to describe the influence of the size of the specimen has been assessed by considering three cubic specimens with 100, 50 and $10 \mathrm{~mm}^{3}$. The two-dimensional simulations were carried out using, again, plain stress elements and symmetric boundary conditions. The configuration of the mesh and the material properties were kept constant for the three specimens. In this way, the results can be directly compared without the effect of randomness. The stress-strain diagrams obtained are given in Figure 9. From the results it can be concluded that the micro-model is able to predict the size-effect behaviour of elements under compression. This feature will have to be taken into account in the calibration of the materials for the simulations performed in Section 4.

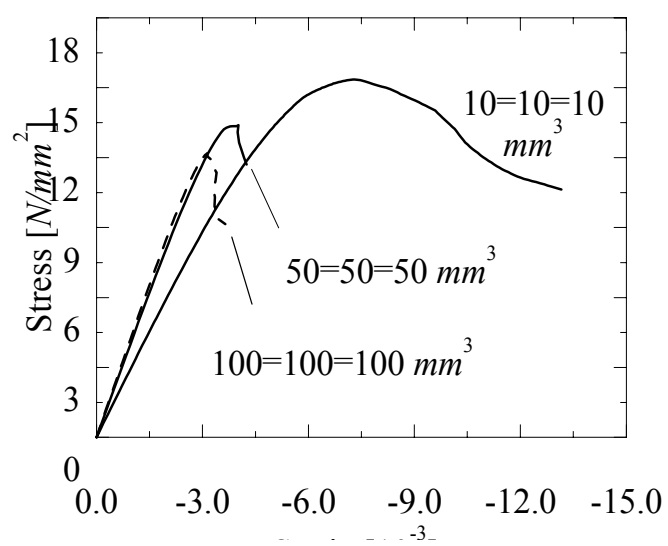

Figure 9: Stress-strain diagrams obtained for different specimen sizes $(R L=2 n$ and $D F=0.3)$. 


\section{Simulation of masonry prisms loaded in compression}

\subsection{Experimental results}

Binda et al [12] carried out deformation controlled tests on masonry prisms with dimensions of $600 \times 500 \times 250 \mathrm{~mm}^{3}$, built up with nine courses of $250 \times 120 \times 55 \mathrm{~mm}^{3}$ solid soft mud bricks and $10 \mathrm{~mm}$ thick mortar joints. Three different types of mortar have been considered and testing aimed at the evaluation of the mechanical compressive properties of the prisms. The characteristics of the masonry components in terms of the compressive strength $f_{c}$, the flexural tensile strength $f_{f}$, the elastic modulus $E$ and the coefficient of Poisson $v$ are given in Table 6. The results obtained for the prisms are given in Table 7. Prisms P1, P2 and P3 were built with mortars M1, M2 and M3, respectively.

\begin{tabular}{|c|c|c|c|c|}
\hline Component & $\begin{array}{c}f_{c} \\
{\left[\mathrm{~N} / \mathrm{mm}^{2}\right]}\end{array}$ & $\begin{array}{c}f_{f} \\
{\left[N / \mathrm{mm}^{2}\right]}\end{array}$ & $\begin{array}{c}E \\
{\left[\mathrm{~N} / \mathrm{mm}^{2}\right]}\end{array}$ & $\begin{array}{c}v \\
{[-]}\end{array}$ \\
\hline Brick & 26.9 & 4.9 & 4865 & 0.09 \\
\hline Mortar M1 & 3.2 & 0.9 & 1178 & 0.06 \\
\hline Mortar M2 & 12.7 & 3.9 & 5648 & 0.09 \\
\hline Mortar M3 & 95.0 & 15.7 & 17758 & 0.12 \\
\hline
\end{tabular}

Table 6: Mechanical properties of the masonry components.

\begin{tabular}{|c|c|c|c|}
\hline Prism type & Mortar type & $\begin{array}{c}f_{c} \\
{\left[N / \mathrm{mm}^{2}\right]}\end{array}$ & $\begin{array}{c}E \\
{\left[N / \mathrm{mm}^{2}\right]}\end{array}$ \\
\hline P1 & M1 & 11.0 & 1651 \\
\hline P2 & M2 & 14.5 & 3833 \\
\hline P3 & M3 & 17.8 & 4567 \\
\hline
\end{tabular}

Table 7: Mechanical properties of the masonry prisms.

\subsection{Numerical results}

The simulations were carried out using a basic cell, i.e., a periodic pattern associated to a frame of reference, see Figure 10 . To reduce computational effort only a quarter of the basic cell was modeled assuming again macro homogeneous symmetry conditions, see Figure 11. A distortion factor equal to 0.3 was assumed for the particles and plain stress conditions under monotonic loading were considered. The dimensions of the components are equal to the experimental ones.

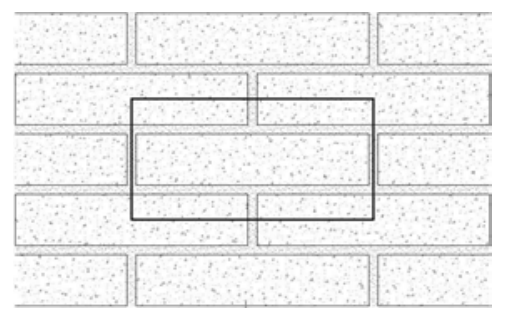

(a)

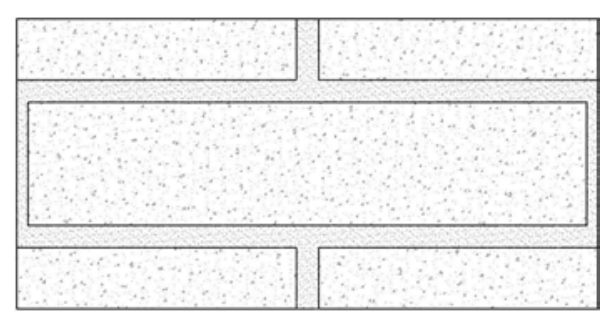

(b)

Figure 10: Definition of basic cell: (a) running bond masonry and (b) basic cell. 


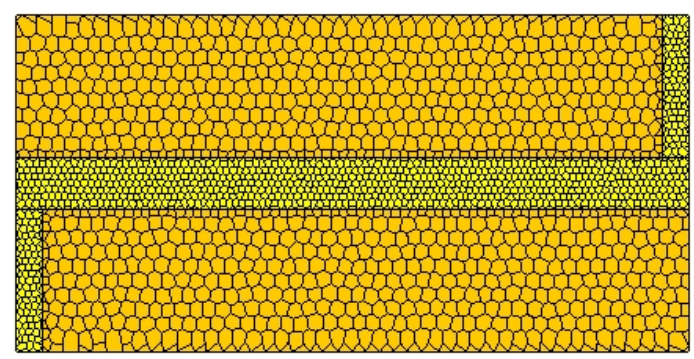

Figure 11: Model used in the single-leaf walls simulations (a quarter of the basic cell, assuming macro homogeneous symmetry conditions).

\subsubsection{Definition of the model parameters from experimental tests}

The micro-model parameters were defined by comparing the experimental and numerical response of the units and mortar considered separately. Each material was modeled resorting to a specimen with the same mesh refinement and distortion factor as the ones used in the composite model (basic cell). Due to the size effect discussed in Section 3.3, the specimens dimensions were assumed equal to the height of the unit and to the thickness of the mortar in the composite model, i.e. $27.5 \times 27.5 \times 27.5 \mathrm{~mm}^{3}$ and $10 \times 10 \times 10 \mathrm{~mm}^{3}$, respectively. Given the stochastic nature of the model, five simulations were performed for each masonry component assuming equal average values for the model parameters.

The aspects related with the definition of the model parameters are now addressed. The values adopted for the interfaces tensile strength $f_{t}$ are slightly lower than the specimens tensile strength, given the contribution of the interfaces shear strength due to the irregular fracture plane. The cohesion $c$ was taken, in general, equal to $1.5 f_{t}$. This is the value recommended for unit-mortar interfaces e.g. by Lourenço [7]. However, quite low experimental ratios between compressive strength and tensile strength were reported from the experimental tests in brick and mortars here considered, with values ranging between four and eight. Due to this reason, cohesion values lower than $1.5 f_{t}$ had to be adopted for Mortar 1 and Mortar 3 .

The values for the friction coefficient $\tan \phi$ were taken so that the numerical compressive strength showed a good agreement with the experimental one. For the mode I fracture energy $G_{f I}$ and mode II fracture energy $G_{f I I}$, experimental values were not available for the materials under analysis and reasonable values had to be assumed. For $G_{f I}$ the values adopted were based in the recommendations proposed in the Model Code 90 [13] for concrete and, also, in the values obtained from the experimental program carried out by Van der Pluijm [8] on masonry components. For $G_{f I I}$, values can also be found in literature for unit-mortar interfaces and a value five times the one recommended by Lourenço [7] for unit-mortar interfaces was assumed, i.e. $0.5 \mathrm{c}$. However, given the exceptional strength of Mortar 3, a lower value equal to $0.3 c$ was adopted.

The parameter values obtained with this procedure are given in Table 8 and, for such input, the response of the model is given in Table 9. In addition, typical numerical stress-strain diagrams obtained for both units and mortar elements are illustrated in Figure 12. 


\begin{tabular}{|c|c|c|c|c|c|}
\hline & & Brick & M1 & M2 & M3 \\
\hline \multirow{4}{*}{ Particles } & $E\left[\mathrm{~N} / \mathrm{mm}^{2}\right]$ & $6000(30 \%)$ & $1500(30 \%)$ & $7000(30 \%)$ & $22000(30 \%)$ \\
\cline { 2 - 6 } & $v[-]$ & $0.094(0 \%)$ & $0.057(0 \%)$ & $0.086(0 \%)$ & $0.115(0 \%)$ \\
\hline \multirow{5}{*}{ Interfaces } & $k_{n}\left[\mathrm{~N} / \mathrm{mm}^{3}\right]$ & $1 \times 10^{4}(0 \%)$ & $104(0 \%)$ & $3 \times 104(0 \%)$ & $8 \times 10^{4}(0 \%)$ \\
\cline { 2 - 6 } & $k_{s}\left[\mathrm{~N} / \mathrm{mm}^{3}\right]$ & $1 \times 104(0 \%)$ & $104(0 \%)$ & $3 \times 104(0 \%)$ & $8 \times 10^{4}(0 \%)$ \\
\cline { 2 - 6 } & $f_{t}\left[\mathrm{~N} / \mathrm{mm}^{2}\right]$ & $3.40(45 \%)$ & $0.75(45 \%)$ & $3.50(45 \%)$ & $10.50(45 \%)$ \\
\cline { 2 - 6 } & $G_{f I}\left[\mathrm{~N} / \mathrm{mm}^{2}\right]$ & $0.170(45 \%)$ & $0.038(45 \%)$ & $0.175(45 \%)$ & $0.525(45 \%)$ \\
\cline { 2 - 6 } & $c\left[\mathrm{~N} / \mathrm{mm}^{2}\right]$ & $5.10(45 \%)$ & $0.30(45 \%)$ & $0.70(45 \%)$ & $15.75(45 \%)$ \\
\cline { 2 - 6 } & $G_{f I I}\left[\mathrm{~N} / \mathrm{mm}^{4}\right]$ & $2.55(45 \%)$ & $0.15(45 \%)$ & $0.35(45 \%)$ & $3.15(45 \%)$ \\
\cline { 2 - 6 } & $\tan \phi[-]$ & $0.10(45 \%)$ & $0.00(0 \%)$ & $0.00(0 \%)$ & $0.10(45 \%)$ \\
\hline
\end{tabular}

Table 8: Values assumed for the model parameters (values in brackets give the $C V$ ).

\begin{tabular}{|c|c|c|c|c|}
\hline & Brick & Mortar 1 & Mortar 2 & Mortar 3 \\
\hline$n[-]$ & 5 & 5 & 5 & 5 \\
\hline$f_{c}\left[\mathrm{~N} / \mathrm{mm}^{2}\right]$ & $27.2(2.7 \%)$ & $3.2(5.0 \%)$ & $12.7(5.4 \%)$ & $95.8(4.4 \%)$ \\
\hline$f_{t}\left[\mathrm{~N} / \mathrm{mm}^{2}\right]$ & $3.61(1.4 \%)$ & $0.64(4.7 \%)$ & $2.70(4.2 \%)$ & $11.62(6.6 \%)$ \\
\hline$E\left[\mathrm{~N} / \mathrm{mm}^{2}\right]$ & $4786(1.9 \%)$ & $1309(1.4 \%)$ & $5632(3.0 \%)$ & $17176(3.1 \%)$ \\
\hline
\end{tabular}

Table 9: Values obtained for the masonry components (values in brackets give the $C V$ ).

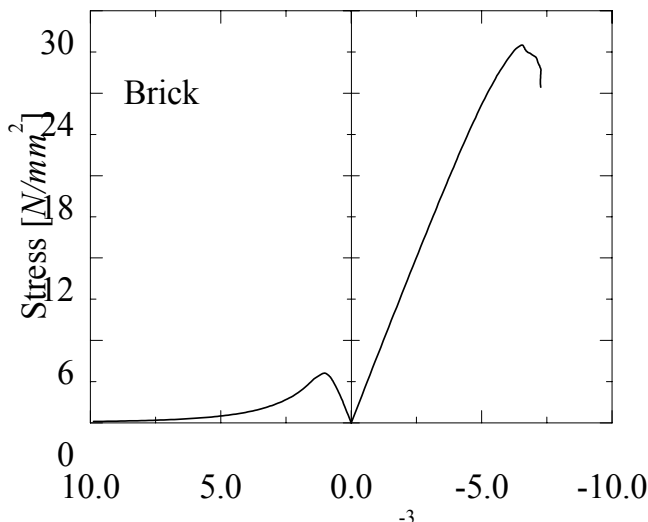

(a)

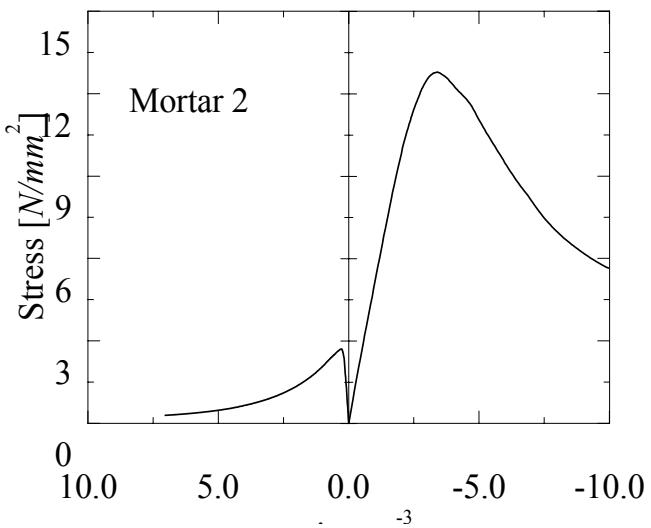

(c)

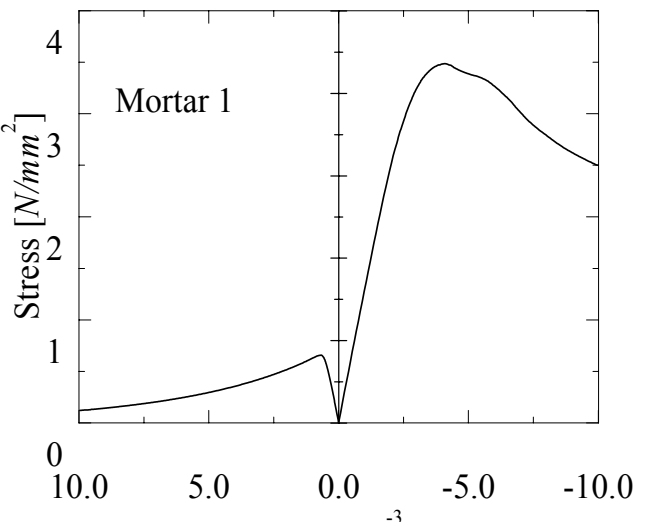

(b)

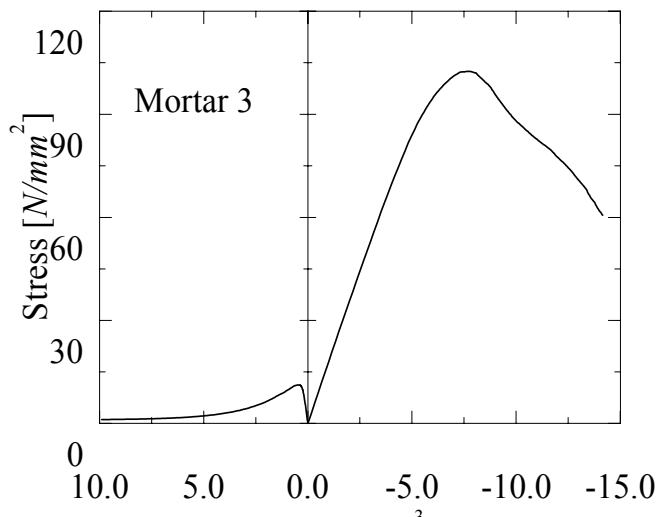

(d)

Figure 12: Typical numerical stress-strain diagrams obtained for the masonry components: (a) brick, (b) Mortar 1, (c) Mortar 2 and (d) Mortar 3. 


\subsubsection{Numerical results and comparison with experimental data}

The compression simulations were carried out on the basic cell and the model parameters given in the previous section were assumed. Only a quarter of the basic cell was modelled due to symmetry reasons but the entire basic cell will be shown in the post-processing results to obtain more legible figures. The model is composed by approximately 13000 linear triangular continuum elements, 6000 linear line interface elements and 15000 nodes. The nonlinear system of equations which follows from the finite element discretization was solved with an incrementaliterative globally convergent Newton-Raphson method with arclength control and line search technique.

The comparison between experimental and obtained numerical results is given in Table 10, where $n$ is the number of specimens. The stress-strain diagrams obtained are shown in Figures 13 and 14. Additionally, the strength values and diagrams obtained from simulations using a non-linear continuum model are also given for a comparison, see [1] for a comprehensive description.

\begin{tabular}{|c|c|c|c|}
\hline & Experimental & Continuum model & Interface model \\
\hline$n[-]$ & 3 & - & 3 \\
\hline Prism 1 & 11.0 & 19.8 & $15.5(2.5 \%)$ \\
\hline Prism 2 & 14.5 & 24.2 & $19.3(3.3 \%)$ \\
\hline Prism 3 & 17.8 & 31.0 & $30.8(2.9 \%)$ \\
\hline
\end{tabular}

Table 10: Experimental and numerical results obtained with the interface model (values in brackets give the coefficient of variation).

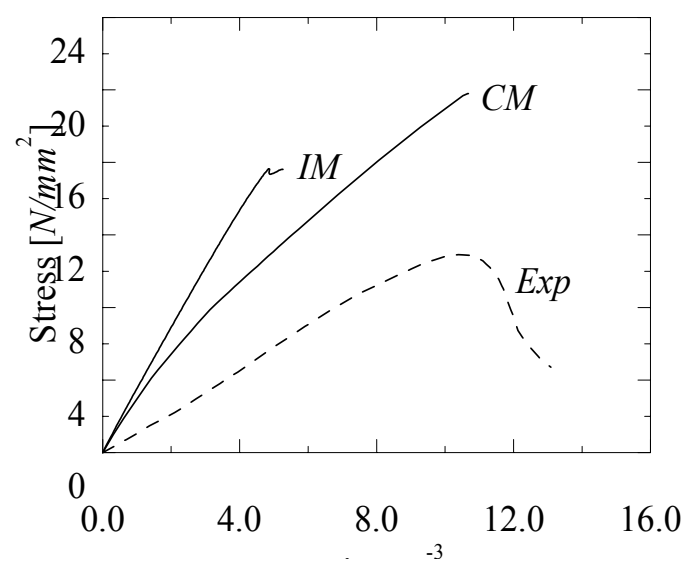

(a)

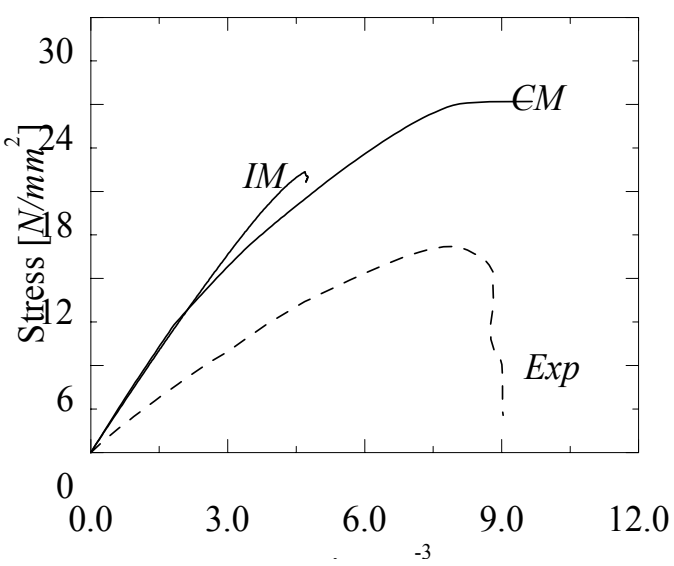

(b)

Figure 13: Numerical and experimental stress-strain diagrams, using experimental mortar stiffness values for: (a) Prism 1 and (b) Prism 2. In the diagrams, $C M$ stands for continuum model, IM stands for the discontinuous interface model (here proposed), and Exp for experimental data. 


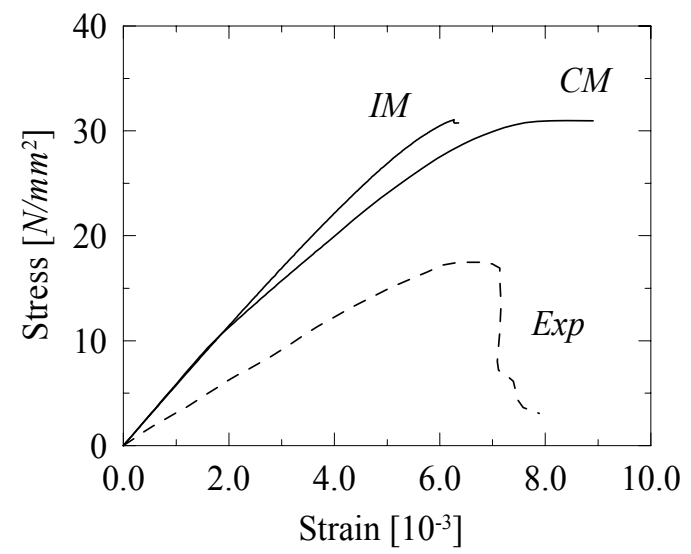

Figure 14: Numerical and experimental stress-strain diagrams, using experimental mortar stiffness values for Prism 3. In the diagrams, $C M$ stands for continuum model, IM stands for the discontinuous interface model (here proposed), and Exp for experimental data.

From the stress-strain diagrams, it is clear that the experimental collapse load is overestimated by the discontinuous interface model. Values of $141 \%, 133 \%$ and $173 \%$ were found for the ratio between the predicted strength and the experimental strength for Prism 1, Prism 2 and Prism 3, respectively. A similar behaviour is shown by the continuum model, although more satisfactory results have been achieved with the interface model. An enormous over-prediction of stiffness has also been found when using the experimental mortar stiffness values. This point will be addressed later in the text.

The failure patterns are an important feature when assessing numerical models and are depicted in Figure 15 for the interface model. The numerical failure patterns found agree reasonably well with typical compression experimental patterns. For the masonry prism built with low strength mortar (Prism 1), localized damage developed at the centre of the units and diffused damage was present for strong mortar masonry (Prisms 2 and 3). Yet, a certain mesh dependence has been shown by the model, expressed by the development of diagonal damage. It has been shown that this deficiency can be overcome by using high geometrical heterogeneity and by taking the discretization of the continuum to a more refined level. Of course, this has the drawback of increasing dramatically the number of mesh nodes and the computational time. It is further noted that in the case of Prism 1, the two cracks found at the centre of the units represent, in reality, a single crack, given the fact that the symmetry conditions adopted and the size and shape of the particles utilized influence the results obtained. 


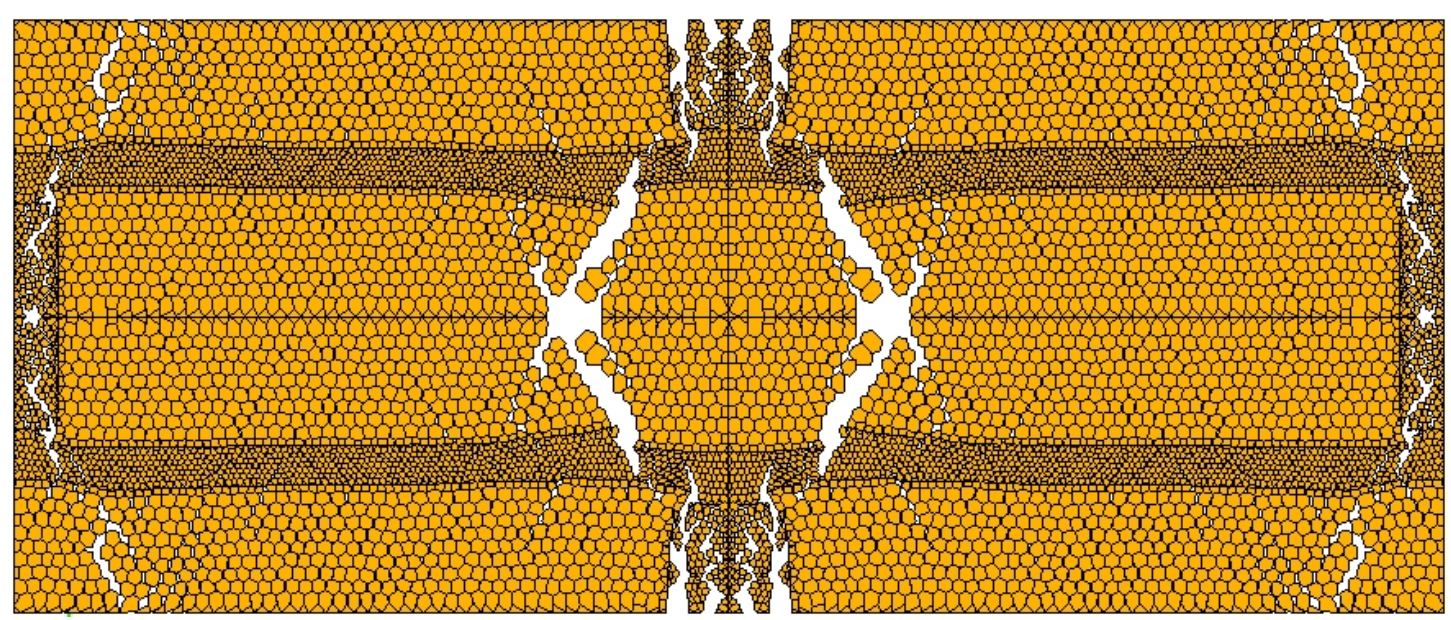

(a)

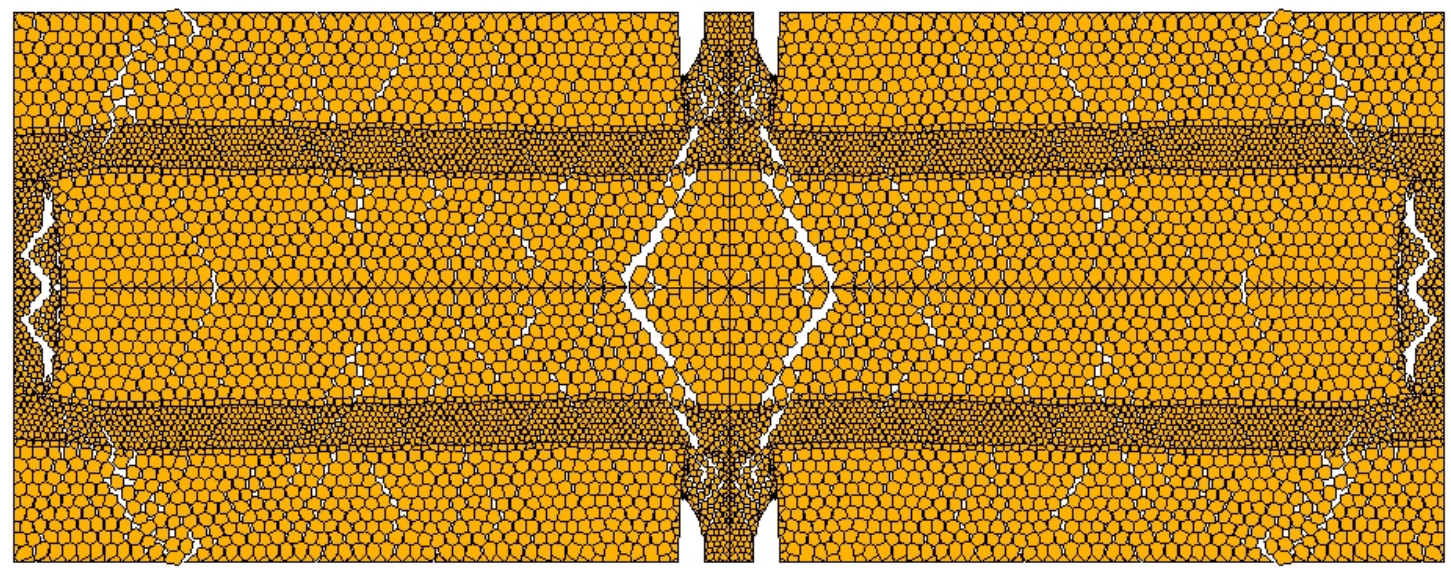

(b)

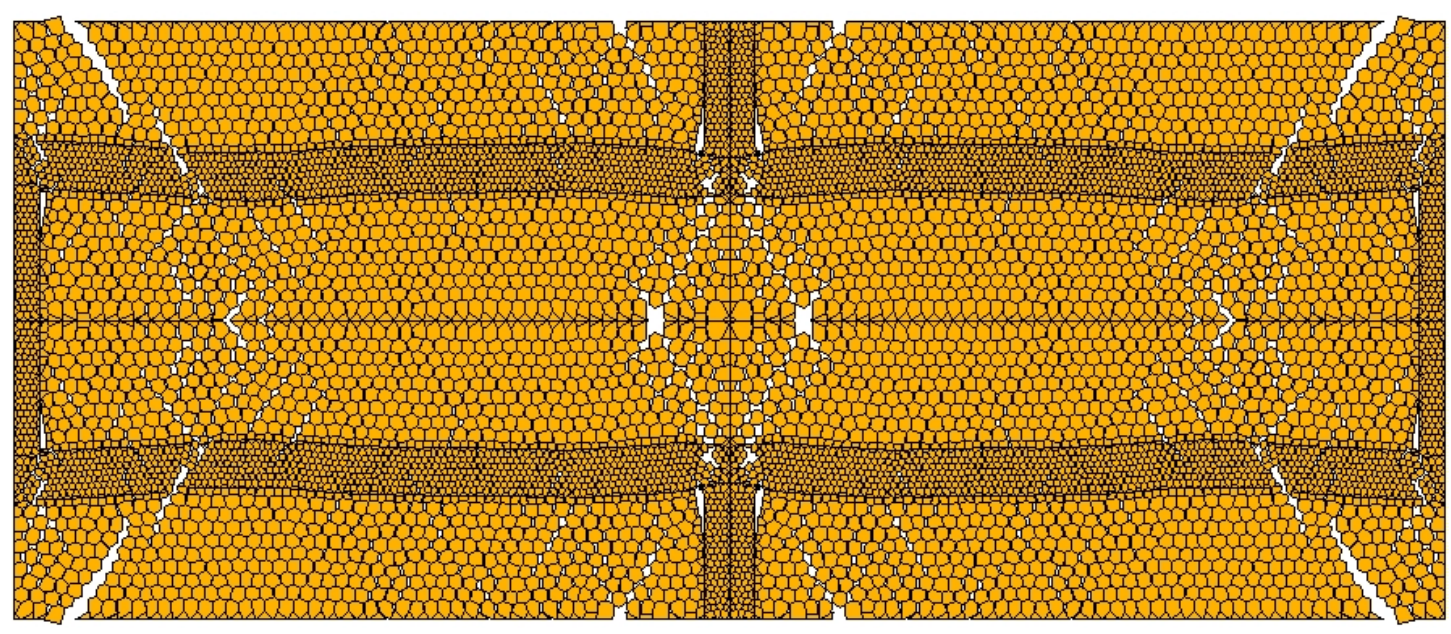

(c)

Figure 15 - Deformed (incremental) meshes near failure: (a) Prism 1,

(b) Prism 2 and (c) Prism 3. 
To reproduce correctly the experimental elastic stiffness of the masonry prisms, the elastic modulus of the mortar must be adjusted. The numerical overestimation of the experimental stiffness is due to mortar laying and curing. The fact that mortar inside the composite is different from mortar specimens cast separately represents a severe drawback of detailed micro-models. Thus, new analyses are necessary, adjusting the elastic stiffness of mortar by inverse fitting, so that deformability properties of the composite are reproduced. An estimate for the value of the adjusted stiffness can be obtained from

$$
\Delta_{y, M}=\Delta_{y, u}+\Delta_{y, m}
$$

where $\Delta_{y, M}$ is the vertical displacement of a masonry prism, $\Delta_{y, u}$ is the vertical displacement contribution of the units and $\Delta_{y, m}$ is the vertical displacement contribution of the mortar joints. This equation reads, after some manipulation,

$$
E_{m}=\frac{h_{m} E_{M} E_{u}}{E_{u}\left(h_{m}+h_{u}\right)-E_{M} h_{u}}
$$

here, $E_{m}$ is the adjusted elastic modulus of the mortar, $E_{u}$ is the elastic modulus of the units, $E_{M}$ is the elastic modulus of the composite, $h_{m}$ is the joint thickness and $h_{u}$ is the height of the unit. The adjusted elastic deformability parameters assumed in the new simulations are given in Table 11.

\begin{tabular}{|c|c|c|c|}
\hline & Particles & \multicolumn{2}{|c|}{ Interfaces } \\
\cline { 2 - 4 } & $E\left[\mathrm{~N} / \mathrm{mm}^{2}\right]$ & $k_{n}\left[\mathrm{~N} / \mathrm{mm}^{3}\right]$ & $k_{s}\left[\mathrm{~N} / \mathrm{mm}^{3}\right]$ \\
\hline Mortar 1 & 350 & $10^{4}$ & $10^{4}$ \\
\hline Mortar 2 & 750 & $10^{4}$ & $10^{4}$ \\
\hline Mortar 3 & 1200 & $10^{4}$ & $10^{4}$ \\
\hline
\end{tabular}

Table 11: Adjusted elastic deformability parameters for mortar.

Due to the very high ratio between the unit and mortar elastic moduli, the iterative solution procedure of the equilibrium equations exhibited severe convergence problems and very small load increments had to be applied. The results obtained with the adjusted stiffness are given in Table 12, together with the results obtained with the experimental stiffness for a better comparison. The stress-strain diagrams obtained are illustrated in Figure 16.

With the adjusted stiffness, a much better agreement between numerical and experimental values for the peak strain was found for the interface model than for the continuum model. Yet, the behaviour of the interface model is more brittle than the experimental one. Both for the interface and the continuum model, the experimental strength of the prisms is overestimated. However, a much better agreement in terms of collapse loads was found with the discontinuous model. It is further noted that the models behave differently when using the experimental mortar stiffness or the adjusted stiffness. While the continuum model yields almost equal 
strength predictions despite of the mortar stiffness, the interface model is sensitive to this parameter and important strength reductions are obtained when adjusted (lower) mortar stiffnesses are assumed, except for Prism 1 built with a weak mortar.

\begin{tabular}{|c|c|c|c|c|c|}
\hline & \multirow{2}{*}{$\begin{array}{l}\text { Experimental } \\
\text { strength }\end{array}$} & \multicolumn{2}{|c|}{ Continuum model } & \multicolumn{2}{|c|}{ Interface model } \\
\hline & & $E_{\text {exp }}$ & $E_{a d j}$ & $E_{\text {exp }}$ & $E_{a d j}$ \\
\hline Prism 1 & 11.0 & $\begin{array}{c}19.8 \\
(180 \%)\end{array}$ & $\begin{array}{c}18.6 \\
(169 \%)\end{array}$ & $\begin{array}{c}15.5 \\
(141 \%)\end{array}$ & $\begin{array}{c}15.4 \\
(140 \%)\end{array}$ \\
\hline Prism 2 & 14.5 & $\begin{array}{c}24.2 \\
(167 \%)\end{array}$ & $\begin{array}{c}24.2 \\
(167 \%)\end{array}$ & $\begin{array}{c}19.3 \\
(133 \%)\end{array}$ & $\begin{array}{c}17.3 \\
(119 \%)\end{array}$ \\
\hline Prism 3 & 17.8 & $\begin{array}{c}31.0 \\
(174 \%)\end{array}$ & $\begin{array}{c}30.1 \\
(169 \%)\end{array}$ & $\begin{array}{c}30.8 \\
(173 \%)\end{array}$ & $\begin{array}{c}24.6 \\
(138 \%)\end{array}$ \\
\hline
\end{tabular}

Table 12: Experimental and numerical compressive strength values, considering the experimental $E_{\text {exp }}$ and adjusted $E_{a d j}$ mortar stiffness values. In brackets are given the strength ratios between the numerical and the experimental values.

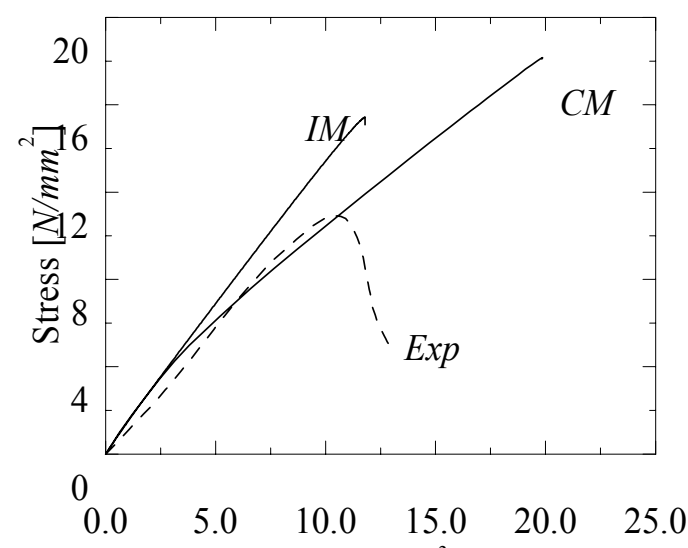

(a)

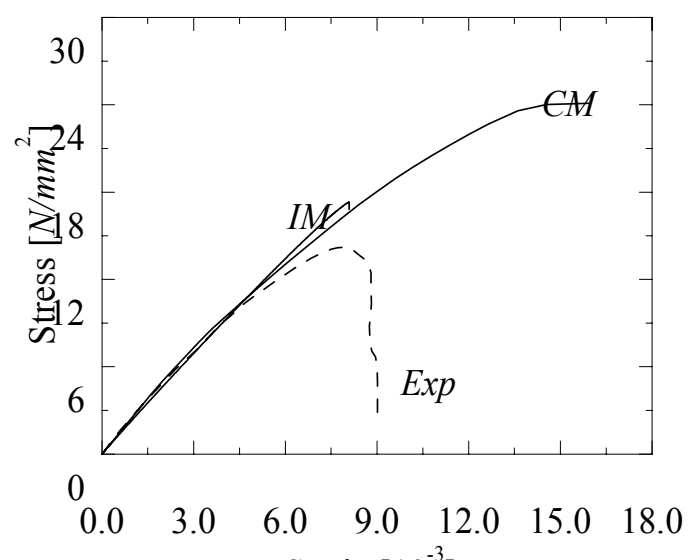

(b)

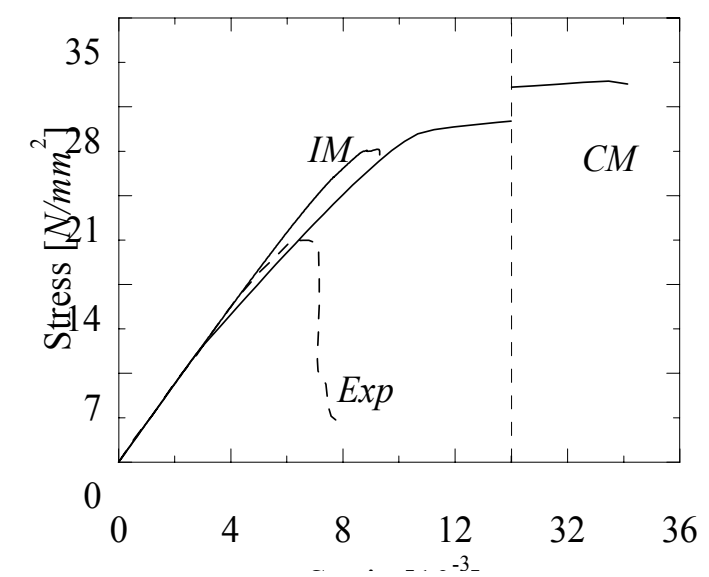

(c)

Figure 16: Numerical and experimental stress-strain diagrams, using adjusted mortar stiffness values: (a) Prism 1, (b) Prism 2 and (c) Prism 3. In the diagrams, $C M$ stands for continuum model, $I M$ stands for interface model, and $\operatorname{Exp}$ for experimental. 


\section{Conclusions}

Continuous and discontinuous approaches have been adopted to represent the microstructure of masonry components, attempting to reproduce the experimental behaviour of masonry under compression. It is noted that the experimental results used for numerical comparison are very demanding with the models, covering a wide range of unit/mortar strength ratios.

The results obtained with short term loading simulations of masonry prisms allow to conclude that: (a) discontinuous models show clear advantages when compared to continuum models in predicting the compressive behaviour of masonry, including strength and deformability properties and (b) shear parameters rather than tensile parameters play a major role at the micro-level and greatly influence the overall response of masonry loaded in compression.

Tentative suggestions for further work are the assessment of other models developed in a discontinuous framework so that reliable estimation of the masonry compressive response can be made.

\section{References}

[1] Pina-Henriques, J., Lourenço, P. B., "Testing and modelling of masonry creep and damage in uniaxial compression", Proc. $8^{\text {th }}$ STREMAH, 2003.

[2] Schellekens, J., "Computational strategies for composite structures", Dissertation, TU Delft, Delft, The Netherlands, 1992.

[3] Lourenço, P. B., "Computational strategies for masonry structures", Dissertation, TU Delft, Delft, The Netherlands, 1996.

[4] DIANA - Diana Finite Element Code, Version 7.2, TNO Building and Construction Research, The Netherlands, 1999.

[5] DIANA - Diana Finite Element Code, Version 8.1, TNO Building and Construction Research, The Netherlands, 2003.

[6] Qhull Version 3.1, Barber, C. and Huhdanpaa, H., University of Minnesota, USA, 2001.

[7] Lourenço, P. B., "A user/programmer guide for the micro-modelling of masonry structures". Report 03.21.1.31.35, TU Delft, The Netherlands, 1996.

[8] Van der Pluijm, R., "Out-of-plane bending of masonry, behaviour and strength", Dissertation, TU Delft, Delft, The Netherlands, 1999.

[9] Schubert, P. "The influence of mortar on the strength of masonry", Proc. $8^{\text {th }}$ IBMaC, London, UK, 162-174, 1988.

[10] Vonk, R., "A micromechanical investigation of softening of concrete loaded in compression", Heron, 38(3), 1993.

[11] Kim, J. and YI, S., "Application of size effect to compressive strength of concrete members", Sadhana, 27(4), 2002.

[12] Binda, L., Fontana, A., Frigerio, G., "Mech. behaviour of brick masonries derived from unit and mortar characteristics", Proc. $8^{\text {th }}$ IBMaC, Ireland, 1988.

[13] CEB-FIP, “CEB-FIP Model code 1990”. Bulletin D'Information no. 213/214, Comite Euro-International du Beton, T Telford, London, UK, 1993. 